\title{
Ultrasound Measurements of Segmental Temperature Distribution in Solids: Method and its High-Temperature Validation
}

\author{
Yunlu Jia, Vasiliy Chernyshev, and Mikhail Skliar \\ Department of Chemical Engineering \\ University of Utah \\ 50 S Central Campus Dr. \\ Salt Lake City, UT 84112
}

\begin{abstract}
A novel approach that uses noninvasive ultrasound to measure the temperature distribution in solid materials is described and validated in high-temperature laboratory experiments. The approach utilizes an ultrasound propagation path with naturally occurring or purposefully introduced echogenic features that partially redirect the energy of an ultrasound excitation pulse back to the transducer, resulting in a train of echoes. Their time of flight depends on the velocity of ultrasound propagation, which changes with temperature distribution in different segments of the propagation path. We reconstruct segmental temperature distributions under different parameterizations. Several parameterizations are discussed, including piecewise constant and piecewise linear, and the parametrization that requires that the estimated temperature profile satisfies an appropriate heat conduction model. The experimental validation of the proposed approach with an alumina sample shows that even with simple parameterizations, the temperature profile is correctly captured with an accuracy that may be comparable to that of the traditional pointwise sensors. The advantages of the approach are discussed, including its suitability for real time and non-destructive temperature measurements in extreme environments and locations inaccessible to the traditional insertion sensors.
\end{abstract}

Keywords: Ultrasound, temperature distribution measurements, high temperature

\section{Introduction}

Velocity of ultrasound propagation in gases, liquids, and solids changes with temperature. Several temperature measurement techniques, such as those reported in [1,2,3], are based on this dependence. For example, an unknown but uniform temperature of a solid material can be obtained in the pulse-echo mode by measuring a delay, $t_{o f}$, in the arrival of an echo reflected from the distal end of the sample relative to the time an elastic excitation pulse was launched into the material by an ultrasound transducer coupled to the proximal end of the sample. If $L$ is the distance between the proximal and the distal ends, then the measurement of the echo's arrival delay (or time of flight, TOF) can be used to calculate the group velocity of the ultrasound propagation (colloquially referred to as the speed of sound), $c$, through the isothermal sample:

$$
c=\frac{2 L}{t_{o f}}
$$

The relationship between the speed of sound and the temperature, $T$,

$$
c=f(T)
$$


then allows us to find the sample's temperature from the measurements of $t_{o f}$ as

$$
T=f^{-1}\left(\frac{2 L}{t_{o f}}\right)
$$

When the temperature of the sample is non-uniform and changes with location, $z$, then the measured time of flight encodes the distribution of the temperature, $T(z)$, along the entire path of the ultrasound propagation according to the following "measurement" equation:

$$
t_{o f}=2 \int_{0}^{L} \frac{1}{f(T(z))} d z
$$

Unlike the unambiguous isothermal case described by equation (3), there are arbitrarily many different distributions $T(z)$ that will result in the same $t_{o f}$ when used in equation (4). If temperature distribution changes with time, it is even possible to have a constant $t_{o f}$ while $T=T(z, t)$ is changing both in time and space.

This non-uniqueness makes the problem of deconvoluting $T(z)$ from the measurement equation (4) ill-posed. System theoretical discussion of similarities of ultrasound and other noninvasive measurement modalities, in which the acquired data depends on the spatial integral of the property of interest, is given in [4]. The problem becomes even more complex if its accurate solution requires that the thermal expansion is explicitly taken into the account, in which case the integration limit itself becomes a function of an unknown temperature distribution: $L=L(T(z))$.

To find an unknown temperature distribution that agrees with the measured $t_{o f}$, additional information or constraints on the admissible solution of equation (4) are needed. Several options may be considered, four of which are outlined below.

Tomographic reconstruction. In this approach, multiple transducers and receivers are used to measure transmit and/or echo delays along different ultrasound propagation paths. The temperature distribution is then reconstructed using a procedure similar to computer tomography (e.g., $[1,5,6])$. Instead or in addition to multiple stationary transducers and receivers, a mobile transducer and/or receiver may be used to scan the domain. For example, Kosugi and Ihara [7] measured the arrival delays of surface acoustic waves created in different locations by a scanned pulsed laser and used the result to estimate the surface temperature of a solid sample.

Regularization. The problem of finding a continuous distribution $T(z)$ is an infinite-dimensional problem often encountered when the state of a spatially distributed system must be estimated [8]. It may be reduced to a finite dimensional problem by "regularization" which amounts to the reduction in the degrees of freedom by imposing additional constraints on the "admissible" solutions. In the regularized form, the functional form of $T(z)$ is prescribed, only parametric variations are allowed, and the solution is obtained by finding unknown parameters using the available ultrasound measurements.

Three different parameterizations of the temperature distribution are considered in this paper. In the simplest case, the temperature non-uniformity is ignored, and its constant value along the path of the ultrasound propagation that agrees with the measured $t_{o f}$ is found. This constant temperature, $T_{a}$, is a single parameter which we can explicitly calculate from equation (3). It gives the uniform temperature needed for the ultrasound echo to arrive with the same delay as was observed with an unknown non-uniform temperature distribution. The relationship of $T_{a}$ to this unknown temperature distribution $T(z)$ is given by the following (generally, nonlinear) integral equation:

$$
2 \int_{0}^{L} \frac{1}{f(T(z))} d z=\frac{2 L}{f\left(T_{a}\right)}
$$

which indicates that $T_{a}$ is generally not equal to the spatially averaged $T(z)$.

In many cases, we expect $T_{a}$ to be a poor approximation of $T(z)$. The approximation is particularly problematic when significant temperature changes are present, as in the case of the temperature distribution across the containments of energy conversion and other high-temperature processes.

An improvement over an assumption of the constant temperature is to assume that the temperature varies linearly between the proximal and distal ends of the sample. This will give not just a better approximation but, in certain cases, the correct result. For example, the temperature is known to change linearly across the walls separating environments maintained at different but constant temperatures. Note, however, that since the linear distribution is defined by two 
parameters (the slope and the intercept), the measurement of $t_{o f}$ alone is not sufficient to reconstruct it. At least one additional independent measurement is required, such the measurement of the temperature at the transducer location.

The third parametrization considered by us requires that, in addition to predicting the correct $t_{o f}$ when substituted in equation (4), $T(z)$ satisfies an appropriately selected heat transfer model and its boundary conditions. Several examples of this approach are found in the literature. Takahashi and Ihara [9] used a one-dimensional heat conduction model to describe the temperature distribution. They assumed that the relationship (2) is linear, and the boundary temperature at the transducer location is known (e.g., independently measured). The temperature of the distal end of the sample was selected as second boundary condition. An alternative boundary condition - the heat flux at the distal end of the sample - was used in [10]. In both cases, the selected distal boundary condition was estimated as the value that minimizes the difference between the TOF predicted by the measurement equation (4) and its measured value. The estimated temperature distribution across the sample is then given by the solution of the heat conduction model with the obtained boundary conditions.

Multiple echoes from each excitation. The third distinct approach is to devise a way to extract more information from each ultrasound excitation than a single time of flight measurement. This is possible if each ultrasound pulse produced multiple ultrasound reflections, caused by echogenic features encountered as it propagates through a sample. In an approach reminiscent to the Bragg grating of optical fibers (see, for example, [11]), Hanscombe and Richards [12] proposed a method in which an ultrasound waveguide is engineered to have regions of periodic ultrasound gratings, each producing echoes with different dominant frequencies determined by grating dimensions. Thermal expansion/contraction change these dimensions and, therefore, shift the frequency content of the echoes. By using gratings of different dimensions at different spatial locations along the ultrasound waveguide, multiple frequency-separated echoes are produced, each encoding temperature changes in different spatial regions. In this approach, the cross-talk between overlapping echoes is reduced, but the sensitivity to the temperature distribution between the grated zones is lost. Furthermore, the accuracy of measurements will suffer when the temperature within each grated zone is spatially varying.

The method for ultrasound measurements of segmental temperature distribution (US-MSTD) [13, 14] is another approach that extracts more than a single TOF data point from each ultrasound excitation. Specifically, it uses the ultrasound propagation path consisting of $n$ segments defined by echogenic features situated along its length. The difference in the time of flight between consecutive echoes, produced by partial ultrasound reflections from these features, is used to estimate the temperature distribution across different segments. In [13], it was assumed that the temperature within each segment is constant and the train of $n$ echoes was used to estimate a piecewise constant approximation of a non-uniform temperature distribution, which varied within a $100{ }^{\circ} \mathrm{C}$ range along a cementitious sample. With such approximation, infeasible step discontinuities in the estimated temperature were present at the boundaries of each segment.

Hybrid approach. Here, the US-MSTD method is extended to use with either the constant or the linearly changing parametrization of the segmental temperature. In the latter case, the discontinuity in the estimated temperature distribution is avoided. The hybrid approach combines the use of multiple echoes from each excitation pulse with the regularization of the temperature distribution within each segment. We demonstrate its performance experimentally by applying it to the measurements of the temperature distribution in an alumina sample maintained at temperatures as high as $1,000{ }^{\circ} \mathrm{C}$. Under both parameterizations, the ability to measure the steady state $T(z)$ and the time varying temperature distribution $T(z, t)$ is demonstrated, and the results are shown to be consistent with independent measurements provided by thermocouples.

\section{Method}

The US-MSTD method is graphically summarized in Fig. 1 for the case when the temperature across the containment of an aggressive process is measured. Its essential components are: a) the structured ultrasound propagation paths with echogenic features creating partial ultrasound reflections from known locations; b) an ultrasound instrumentation needed to create the excitation pulse and receive the train of resulting echoes; c) the signal processing of the ultrasound waveforms needed to accurately determine the speed of sound or its change in different segments of the propagation path by measuring the echoes' time of flight; d) the relationship between the speed of sound and the 


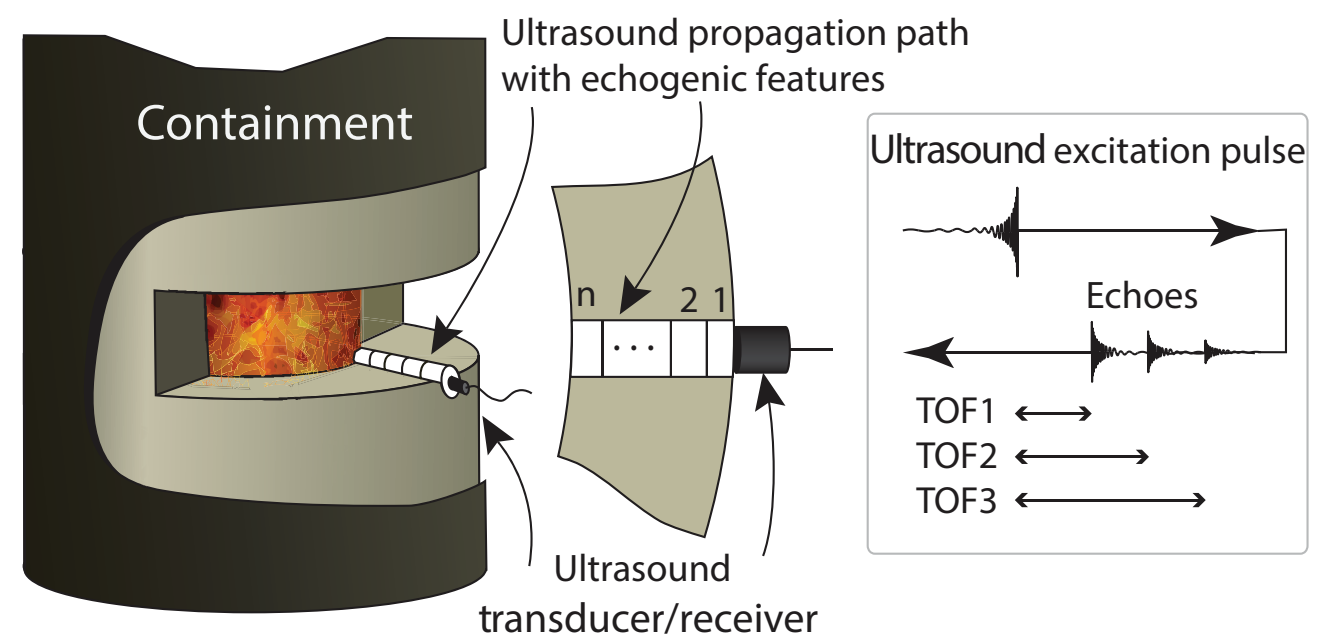

Figure 1: The excitation pulse created by a transducer propagates through the structured containment and encounters $n$ echogenic features along the way, which produce a train of echoes acquired by the receiver. The TOF difference between consecutive echoes is used to estimate the temperature distribution in the corresponding segment of the containment.

temperature; and e) the method to translate the segmental speed of sound into the temperature distribution such that the predicted time of flight, according to the measurement model (4), matches the measured value. In the following, these components are discussed in more detail.

\subsection{Structured ultrasound propagation path}

The train of echoes from each excitation pulse is created by echogenic features located at known locations along the path of the ultrasound propagation. They redirect a fraction of the ultrasound energy back to the transducer where they are recorded and analyzed in order to determined the arrival delay of each echo. The TOF of these echoes encodes the temperature distribution along different segments of the sample. Used together, multiple TOFs provide the data on the temperature distribution along the entire propagation path with finer granularity by providing information specific to the temperature distribution within individual segments.

Several methods for producing partial ultrasound reflections were discussed in [13]. It was found that inclusions, stratifications, and variations in the waveguide geometry are the adequate means in creating the ultrasound propagation path that produce a train of echoes emanating from known spatial locations. The high-temperature experimental demonstration of the US-MSTD method, discussed in this paper, was carried out with echogenic features created by geometric variations introduced by drilling small holes along the length of a ceramic (alumina) waveguide.

An alternative to purposefully designing the waveguide, the echogenic features, occurring naturally or introduced for reasons unrelated to the needs of the US-MSTD method, may also be used. These may include natural stratifications, inclusions, or geometric features, such as a rifling inside a gun barrel used in [10] to estimate the temperature variations caused by firing a gun. It is only required that the spatial locations of echogenic features are characterized prior to their utilization in the US-MSTD method.

\subsection{Acquisition of echo waveforms}

As the ultrasound excitation pulse propagates through the structured material, multiple echoes are produced by each echogenic feature encountered along its path. The waveforms of returned echoes are the primary data used to measure the segmental time of flight and to estimate the temperature distribution in each segment. The return delay $t_{o f}^{z_{i}}$ of an echo produced by a feature located at $z_{i}$ is related to the temperature distribution between the traducer location and $z_{i}$ as

$$
t_{o f}^{z_{i}}=2 \int_{0}^{z_{i}} \frac{1}{f(T(z))} d z
$$


where $z=0$ is the location of the trasducer/receiver. The temperature distribution in the $i$-th segment of the propagation path is inferred from the segmental time of flight, $t_{o f_{i}}$, equal to the difference in the time of flights of consecutive echoes produced by echogenic features at the locations $z_{i}$ and $z_{i-1}$ which bound the segment:

$$
t_{o f_{i}}=t_{o f}^{z_{i}}-t_{o f}^{z_{i-1}}=2 \int_{z_{i-1}}^{z_{i}} \frac{1}{f(T(z))} d z
$$

where $\left(z_{i}-z_{i-1}\right)$ is the segment's length. We often use the first segment between the transducer and the first echogenic feature at $z_{1}$ as a delay line and reference the time of flight of all subsequent echoes to the arrival time of the first echo, $t_{o f}^{z_{1}}$. The difference in the TOF between the second and the first echoes then gives the information on the temperature distribution in the second segment, and so on.

\subsection{Signal processing}

We use signal analysis of the acquired echo waveforms to determine the segmental time of flight. In the simplest case, the arrival delay of an echo may be quantified by timing single-point features on its waveform, such as the first zero crossing or the peak value of the acquired signal. Though standard, these timing techniques are sensitive to measurement noise [15]. Furthermore, when broad-band excitations are used, the timing accuracy of single-feature methods deteriorate further due to waveform distortions and broadening caused by stronger attenuation of higherfrequency content of ultrasound pulses.

A more robust and accurate measurement of $t_{o f}$ and $t_{o f_{i}}$ may be achieved when the entire shape of the waveform is utilized in timing. In this case, both amplitude and phase information are taken into the account [16], which makes timing results less sensitive to measurement noise and shape distortions. The temporal shift needed to obtain the best match between the waveforms may be found by maximizing their cross-correlation [17], minimizing $l_{1}$ and $l_{2}$ norms of their difference [15, 18, 19], or by maximum likelihood [20].

Though cross-correlation and other shape-matching methods perform better than single-point timing, the results may still be unacceptable when a significant distortion of ultrasound waveforms occurs, as is often the case when the pulse propagates through attenuating materials. It was suggested by Le [21] that for waveform-distorting samples, a higher precision can be achieved if the envelopes of the waveforms are used in timing. We have recently shown that further improvements in timing accuracy can be achieved by iteratively applying a nonlinear anisotropic diffusion filter to the envelopes of the echo waveforms [22].

\subsection{Time of flight dependance on temperature}

The time of fight of ultrasound echoes is affected by temperature-dependent changes in the velocity of the ultrasound propagation and the thermal expansion/contraction which changes the length of the ultrasound propagation. These factors typically contribute to the lengthening of an echoes' time of flight as temperature increases. For narrow temperature ranges, the contribution of thermal expansion is negligible. Its importance grows as the range of temperatures across the sample increases. By determining the coefficient of the thermal expansion, $\alpha$, of the material, it is possible to adjust for the change in the length of the ultrasound propagation and thus separate the contributions of this factor to the TOF measurements. As further discussed in sections 3.5 and 4.1, separating the effect of thermal expansion is not always necessary as it is possible to exactly cancel the error it introduces to the estimation of the piecewise-constant temperature distribution.

\subsection{Estimation of temperature distribution}

The segmental time of flight $t_{o f_{i}}$ encodes the information about the temperature distribution within the $i$-th segment. As discussed below, all parametrization options discussed in the context of the measurement model (4) may be used to estimate the segmental temperature distribution from the measurement model (7). 


\subsubsection{Piecewise constant distribution}

This distribution is obtained by assuming constant speed of sound within each segment. When this assumption in used in equation (7), the constant speed of sound in the $i$-th segment of the waveguide is obtained as

$$
c_{i}=\frac{2\left(z_{i+1}-z_{i}\right)}{t_{o f_{i}}}
$$

and the corresponding constant temperature is obtained by inverting the correlation (2). After repeating the process for all segments, the entire temperature distribution along the waveguide is approximated as a piecewise constant function. Such parametrization was used in [13], where it was noted that infeasible temperature discontinuities occurring at the locations of the echogenic features is an undesirable feature of the piecewise constant parameterization. Nevertheless, the experimental results in [13] showed a substantial improvement in accuracy of the piecewise constant approximation compared to the assumption that a constant temperature $T_{a}$ is maintained over the entire path of the ultrasound propagation. Furthermore, by using a larger number of echogenic features, and the correspondingly finer segmentation of the propagation path, the accuracy of this approximation can be improved.

\subsubsection{Piecewise linear distribution}

The temperature continuity at the boundaries of a segment may be enforced by adopting a piecewise linear parametrization of the temperature distribution. In this case, the linear temperature change in the $i$-th segment is parameterized as

$$
T(z)=m_{i} z+n_{i}, \quad z_{i-1} \leq z \leq z_{i}
$$

where $m_{i}$ and $n_{i}$ are unknown coefficients. If we assume that the temperature $T\left(z_{0}\right)$ at the transducer location $z_{0}=0$ is independently measured, then the distribution in the first segment is defined by an unknown slope $m_{1}$. Using the measurement of the first segmental time of flight, we can find an unknown $m_{1}$ from the following spacial case of equation (7):

$$
t_{o f_{1}}=2 \int_{0}^{z_{1}} \frac{1}{f\left(m_{1} z+n_{1}\right)} d z
$$

Using the measured difference in the TOF of the second and first echoes, the unknown slope $m_{2}$ and intercept $n_{2}$ are obtained from the solution of the following two equations:

$$
\begin{aligned}
t_{o f_{2}} & =2 \int_{z_{1}}^{z_{2}} \frac{1}{f\left(m_{2} z+n_{2}\right)} d z \\
n_{2} & =\left(m_{1}-m_{2}\right) z_{1}+n_{1}
\end{aligned}
$$

where the second equation enforces the continuity of the temperature at $z=z_{1}$. The process is repeated for all remaining segments until the piecewise linear approximation of the temperature distribution over the entire sample is obtained.

\subsubsection{Parametrization with thermal conductivity model}

The temperature parametrization by a one-dimensional heat conduction models was used in references [9] and [10]. In both cases, the temperature of the distal end was unknown and elevated, the temperature at the location of the transducer was assumed to be independently measured, and the temperature distribution was estimated by adjusting a single boundary condition in order to match the predicted and the measured TOF of an echo produced by a reflection of the excitation pulse from the distal end of the ultrasound propagation path. When a two- or three-dimension model is needed to provide an adequately accurate description of the temperature distribution in the sample, additional measurements will be required to reconstruct the temperature distribution. For example, consider the case of a cylindrical waveguide. Assuming the radial symmetry of the temperature distribution, known constant density $\rho$, heat conductivity $k$ and capacity $C_{p}$, the temperature distribution must satisfy the following $2 \mathrm{D}$ heat transport model in cylindrical coordinates:

$$
\rho C_{p} \frac{\partial T}{\partial t}=k\left(\frac{1}{r} \frac{\partial}{\partial r} r \frac{\partial T}{\partial r}\right)+\frac{\partial^{2} T}{\partial z^{2}}
$$




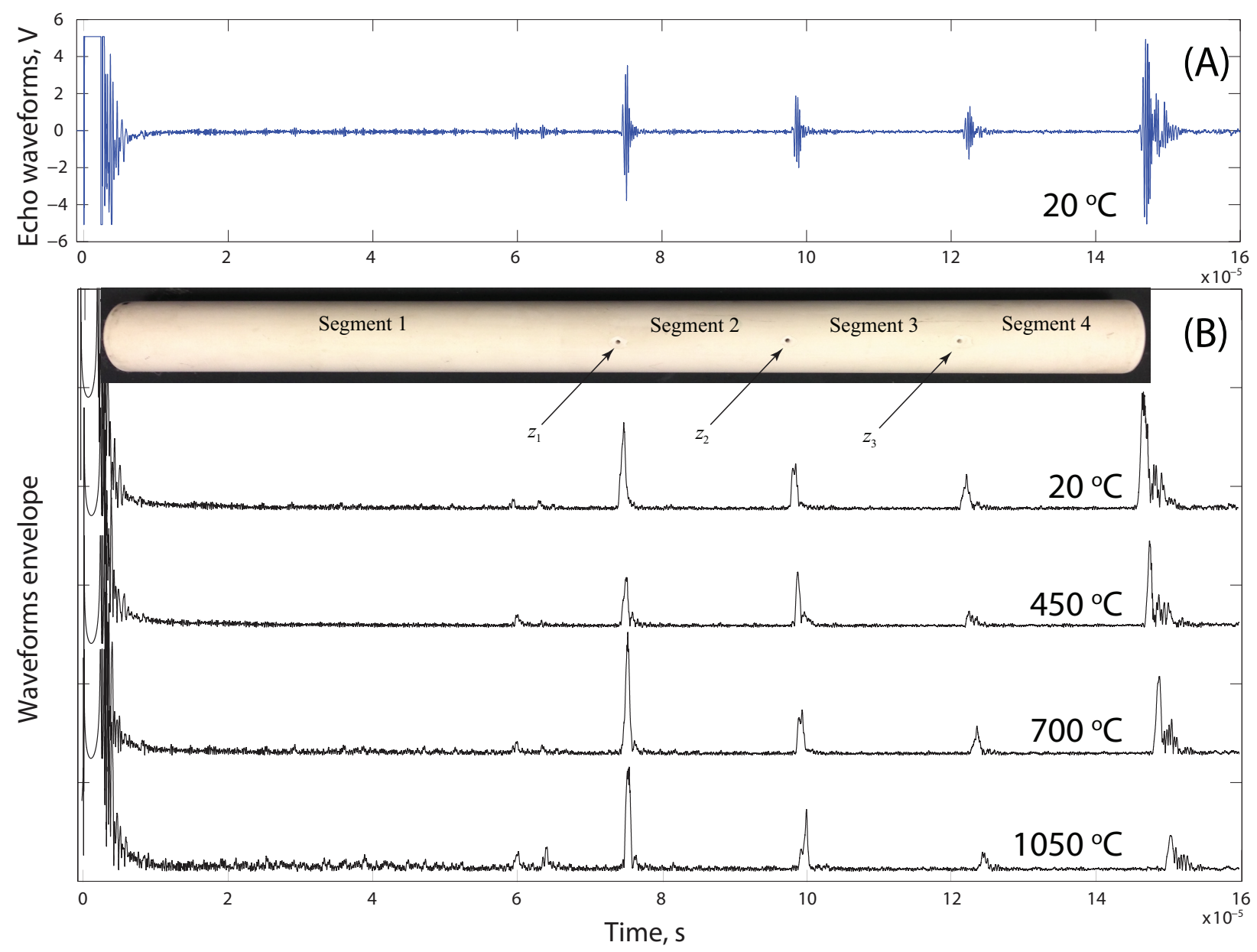

Figure 2: (A) Typical waveforms of ultrasound echoes created by small holes at the locations $z_{i}$, which define different segments of the alumina waveguide (insert). The last echo is created by the reflection from the distal alumina-air interface. The shown waveforms were acquired at the reference temperature equal to $20^{\circ} \mathrm{C}$. (B) Envelopes of echo waveforms acquired at different temperatures.

where $r$ is the radial position relative to the centerline of the sample. To completely define the problem, three boundary conditions - at the proximal, distal and the cylindrical surfaces of the waveguide - are required. If the waveguide is unstructured, only a single ultrasound echo will be produced by a reflection from a distal end of the sample and measurement of its TOF will allow us to estimate only one of the three needed boundary conditions. The other two boundary conditions must be obtained from independent measurements. For example, if the temperatures of the distal and proximal ends of the waveguide are independently measured, then the measured $\Delta t_{o f}$ can be used to estimate the overall heat transfer coefficient $h$ and define the remaining boundary condition given as the heat flux through the cylindrical boundary of the waveguide:

$$
q=h\left(T_{e}-T\right)
$$

where $T_{e}$ is the ambient temperature of the environment. The time of flight of multiple echoes received when the excitation pulse propagates through a structured waveguide may provide sufficient data to estimate all required boundary conditions without the need for additional independent measurements. When such independent measurements are available, they can still be incorporated into the US-MSTD method and may help improve the accuracy and the robustness of the estimated temperature distribution. 
Table 1: Location of echogenic features, $z_{i}(\mathrm{~cm})$, at the reference temperature.

\begin{tabular}{l|c}
\hline$z_{0}$ (proximal end) & 0 \\
$z_{1}$ & 15.36 \\
$z_{2}$ & 20.35 \\
$z_{3}$ & 25.3 \\
$z_{4}$ (distal end) & 30.48
\end{tabular}

\section{Experimental demonstration}

\subsection{Waveguide}

In this study, a ceramic rod (12" long and 1" in diameter; $L=0.3048 \mathrm{~m}, 0.0254 \mathrm{~m}$ OD) pressure-formed from high-purity alumina (Rescor 960 Alumina, Cotronics Corp., Brooklyn, NY) and rated by the manufacturer for continuous operation at temperatures up to $1,650^{\circ} \mathrm{C}$ was used as a waveguide. Echogenic features along its length were introduced by drilling three radially-oriented 3/32"-diameter holes all the way through the rod. The insert in Fig. 2 is the photograph of the actual waveguide, showing the drilled holes. As a first step in precisely determining their axial positions, $z_{1}, z_{2}$, and $z_{3}$, the velocity of the ultrasound propagation inside the waveguide at the reference temperature $T_{r e f}=20^{\circ} \mathrm{C}$ was calculated from equation (4), where $t_{o f}=t_{o f}^{z_{4}}$ is the time of flight of the echo reflected from the distal end of the sample (the last echo in Fig. 2A) and $L$ is the length of the waveguide measured by a micrometer (equal to the axial coordinate of the distal end of the waveguide, $z_{D E}=z_{4}$ ). Using the found value of $c$ and the measurements of the time of flight of the three echoes produced by echogenic features, their coordinates were then found as $z_{i}=c t_{o f}^{z_{i}}$. Table 1 summarizes the results of the described localization procedure. The segments $L_{1}, L_{2}, L_{3}$, and $L_{4}$ are defined by the proximal and distal ends of the waveguide $\left(z_{0}\right.$ and $\left.z_{4}\right)$ and the echogenic features. Using the values listed in Table 1, their lengths were calculated as $L_{i}=z_{i}-z_{i-1}$. Note that $\sum_{i=1}^{4} L_{i}=L$.

\subsection{Ultrasound excitation}

The frequency of the ultrasound excitation must be selected to balance the achievable spatial resolution of the US-MSTD method and the strength of the returned echoes. Echogenic features of small dimensions are needed to achieve finer segmentation of the ultrasound propagation path. Their small size requires a higher-frequency ultrasound excitation in order to avoid diffraction of the propagating elastic waves around small features. However, high frequency ultrasound is more strongly attenuated in solids, which negatively impacts the signal-to-noise ratio in the acquired echo waveforms and limits the length over which the temperature distribution can be estimated.

To find an appropriate tradeoff between these two factors, four transducers (Panametrics model V601, V603, V609, and V611; Olympus IMS, Waltham, MA) with central frequencies equal to $0.5,1,5$, and $10 \mathrm{MHz}$ were tested with the described waveguide. Typical echo waveforms from the three echogenic features and the distal end of the sample for different excitation frequencies are shown in Fig. 3. Using the measured velocity of the ultrasound propagation in the alumina sample (found to be approximately $4,200 \mathrm{~m} / \mathrm{s}$ at room temperature), the wavelength $\lambda=c / f$ of the 0.5 $\mathrm{MHz}$ excitation is $\sim 0.021 \mathrm{~m}$, which is close to the diameter of a 3/32" $(0.024 \mathrm{~m})$ hole. The dimension of echogenic features is comparable with the ultrasound wavelength, which causes the wave diffraction and explains the absence of clear echo signals when $0.5 \mathrm{MHz}$ transducer was used (Fig. 3A). The ultrasound excitation produced by the remaining three transducers produces the expected echoes. The cleanest response was seen with 5 and $10 \mathrm{MHz}$ transducers (Fig. $3 \mathrm{C}$ and $\mathrm{D})$. The attenuation of the higher frequency $(10 \mathrm{MHz})$ excitation is noticeably stronger, resulting in weaker echoes. Therefore, in the subsequent experiments the $5 \mathrm{MHz}$ transducer was used.

\subsection{Speed of sound at different temperatures}

The correlation between the speed of sound and the temperature was established for the $L_{4}$ segment of the waveguide (defined by the distal end of the waveguide and the echogenic feature located at $z_{3}$ ), which was placed in the center of the heating zone (colored segment in Figure 4) of the laboratory tube furnace (Carbolite model MTF 201, Newtown, PA), where the temperature is unform. The partially inserted waveguide was held in place without contact with the surface of the furnace, and the ultrasound transducer was coupled to the end of the waveguide, which was extended outside the heating zone. In this arrangement, the segment of the waveguide between the transducer and 

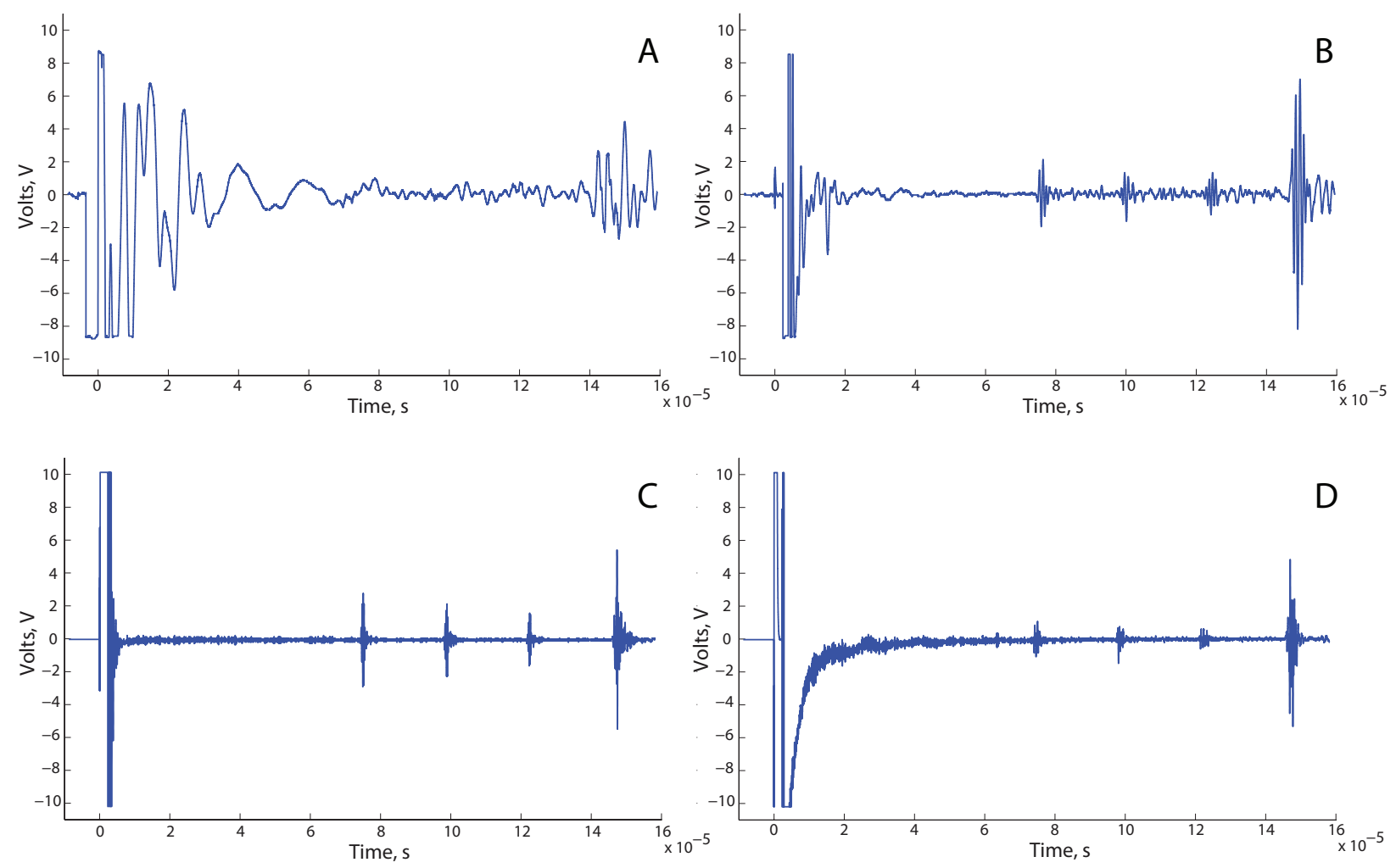

Figure 3: For the experiments conducted at the reference temperature, echo waveforms of the transducers with $0.5,1,5$, and $10 \mathrm{MHz}$ central frequencies are shown in graphs $\mathrm{A}, \mathrm{B}, \mathrm{C}$, and D, respectively.

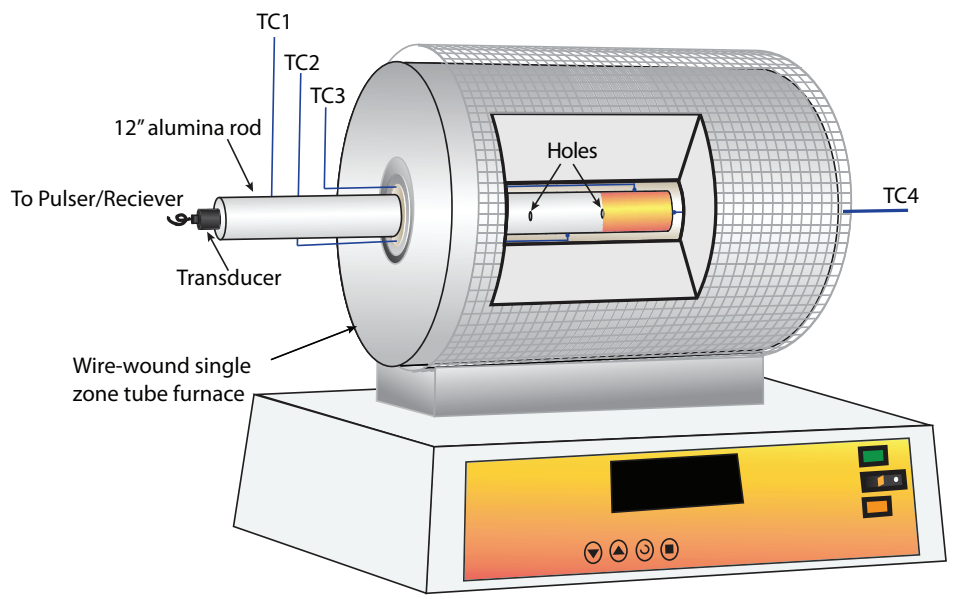

Figure 4: The calibration curve establishing the dependence of the speed of sound on temperature was obtained experimentally for the $L_{4}$ segment of the waveguide, which was placed in the middle of the tube furnace. To validate the US-MSTD method, a similar setup was used; however, only the 4th segment of the waveguide was inserted into the furnace, with the remaining segments extending outside the heating zone. 
the echogenic feature located at $z_{3}$ is effectively an ultrasound delay line over which the heat dissipated to ensure that the temperature of the waveguide's proximal end is below the Curie point of the traducer. Four Nextel ceramic insulated thermocouples (OMEGA Engineering, Inc., Stamford, CT) were attached to the surface of the waveguide with a ceramic adhesive and provided independent temperature measurements during the experiments. Three thermocouples (TC) were attached to the cylindrical surface of the waveguide at approximate axial locations equal to $z_{i}+\left(z_{i+1}-z_{i}\right) / 2, i=1, \ldots, 3$, which are the middle points between the echogenic features. The fourth thermocouple was attached to the distal end of the waveguide (in the middle of the circular surface with an axial coordinate $z_{4}$ ).

During the calibration experiments, the temperature setpoints for the furnace were changed in $50{ }^{\circ} \mathrm{C}$ increments, spanning the range between $50^{\circ} \mathrm{C}$ to $1,150^{\circ} \mathrm{C}$. Each experiment was repeated six times. To avoid potential measurement bias, the sequence of test temperatures, including all repeat experiments, was randomized. After each change in the setpoint, sufficient time was allowed for thermal equilibration to occur before the ultrasound measurements were performed.

The ultrasound measurements were carried out using Panametrics pulser/receiver (model 5072PR; Olympus IMS, Waltham, MA) and Panametrics transducer with a central frequency of $5 \mathrm{MHz}$ (models V609). The waveform data were acquired at $500 \mathrm{MHz}$ sampling rate using Tektronix oscilloscope (Tektronix Inc., Beaverton, OR; model MSO 2024), which was set to report data after internal averaging of 16 waveforms. The oscilloscope was interfaced to a computer running a custom Matlab data acquisition and signal analysis code.

\subsection{Signal processing}

The examination of waveforms in Fig. 2A shows that the shape of echoes that travel longer distances prior to reaching the receiver are broader, more distorted, and attenuated. This is an expected outcome for the dissipative waveguide, like ours, which has a stronger attenuation of higher frequency components of the ultrasound signals. Under these conditions, the timing of ultrasound echoes based on the analysis of their waveforms is susceptible to significant errors [22]. As a more robust alternative, we timed the arrival of ultrasound echoes by cross-correlating their envelopes. To define an envelope of a waveform, $s(t)$, we start by introducing the corresponding analytic signal, $s_{a}(t)$, given as the following complex function of $s(t)$ :

$$
s_{a}(t)=s(t)+j \hat{s}(t)
$$

where $j^{2}=-1$ and $\hat{s}(t)$ is the Hilbert transform of $s(t)$ :

$$
\hat{s}(t)=-\frac{1}{\pi} \lim _{\varepsilon \rightarrow 0} \int_{\varepsilon}^{\infty} \frac{s(t+\tau)-s(t-\tau)}{\tau} d \tau .
$$

The envelope of the waveform $s(t)$ acquired when the sample is maintained at the temperature $T$ is the amplitude of its analytic signal:

$$
A_{T}(t)=\left|s_{a}(t)\right|=\sqrt{s^{2}(t)+\hat{s}^{2}(t)}
$$

The envelopes of the echoes in Fig. 2A are shown as the top graph in Fig. 2B. The remaining graphs in Fig. $2 \mathrm{~B}$ give the envelopes of the echoes collected when the sample was maintained at the elevated uniform temperatures equal to 450,700 , and $1,050^{\circ} \mathrm{C}$. Visual comparison reveals distinct trend towards an increased time of flight as the temperature rises, which corresponds to the reduction in the speed of sound at the elevated temperatures. Prior to formulating the envelope cross-correlation problem which we solve to quantify the impact of the temperature on the speed of sound, the needed timing measurements and their correlation to uniform and non-uniform distributions of the segmental temperature are discussed first.

\subsubsection{Uniform temperature}

We quantify the change in the time of flight of the $i$-th echo, $\Delta t_{o f}^{z_{i}}$, in the waveguide maintained at the uniform temperature $T$ relative to its TOF at the uniform reference temperature, $T_{r e f}=20^{\circ} \mathrm{C}$. For the propagation path of length $z_{i}$ maintained at a uniform temperature $T$ :

$$
\Delta t_{o f}^{z_{i}}(T)=t_{o f}^{z_{i}}\left(T_{r e f}\right)-t_{o f}^{z_{i}}(T)=2\left[\frac{z_{i}}{c\left(T_{r e f}\right)}-\frac{z_{i}}{c(T)}\right]
$$


This result can be used to calculate the change in the speed of sound caused by the temperature transitions from $T_{r e f}$ to $T$ in the segments of the waveguides $L_{1}, \ldots, L_{i}$ between the transducer and $z_{i}$. The change in the speed of sound in segment $L_{i}$ can be found from the measurements of the change in the time of flights, $\Delta t_{o f}^{z_{i-1}}(T)$ and $\Delta t_{o f}^{z_{i}}(T)$, of the two consecutive echoes produced by echogenic features located at $z_{i-1}$ and $z_{i}$ :

$$
c_{i}=\frac{2\left(z_{i}-z_{i-1}\right)}{t_{o f_{i}}(T)}=\frac{2\left(z_{i}-z_{i-1}\right)}{\left(t_{o f_{i}}\left(T_{r e f}\right)-t_{o f_{i-1}}\left(T_{r e f}\right)\right)+\left(\Delta t_{o f}^{z_{i}}(T)-\Delta t_{o f}^{z_{i-1}}(T)\right)} .
$$

\subsubsection{Non-uniform temperature}

When the temperature of the sample is non-uniform, equation (18) does not hold, and the dependance of the measured change in the TOF of $i$-th echo on the temperature distribution $T(z)\left(z \in\left[z_{0}, z_{i}\right]\right)$ relative to the TOF at the uniform reference temperature is given by the following expression:

$$
\Delta t_{o f}^{z_{i}}(T)=2\left[\frac{z_{i}}{c\left(T_{r e f}\right)}-\int_{0}^{z_{i}} \frac{1}{f(T(z))}\right] d z .
$$

The segmental time of flight is related to segmental temperature distribution by the following variant of the measurement equation (7):

$$
t_{o f_{i}}(T)=2 \int_{z_{i-1}}^{z_{i}} \frac{1}{f(T(z))} d z
$$

It is convenient to calculate $t_{o f_{i}}(T)$ as a difference between the TOF of the $(i-1)$-th and $i$-th echoes at the reference condition plus the contribution of the measured change in the TOF due to an unknown temperature distribution $T(z)$ :

$$
t_{o f_{i}}(T)=\left(t_{o f_{i}}\left(T_{r e f}\right)-t_{o f_{i-1}}\left(T_{r e f}\right)\right)+\left(\Delta t_{o f}^{z_{i}}(T)-\Delta t_{o f}^{z_{i-1}}(T)\right)
$$

An unknown temperature distribution $T(z)$ within the segment must now be found by using the discussed options for the temperature parametrization. For example, under piecewise constant parameterization, the segmental speed of sound would be calculated from equation (8) and the corresponding temperature will be found from equation (3).

\subsubsection{Timing ultrasound echoes}

The temperature-induced change in the TOF of ultrasound echoes is determined as the solution of the following optimization problem:

$$
\Delta t_{o f}^{z_{i}}(T)=\operatorname{argmax}_{\tau} \int_{-\infty}^{+\infty} A_{T_{r e f}}(t) A_{T}(t+\tau) d t .
$$

In words, $\Delta t_{o f}^{z_{i}}$ is found as a shift $\tau$ needed to maximize the correlation between the envelopes of the given and the reference echoes. The found value is related to the speed of sound by either equation (18) or (20), depending on whether the sample is maintained at a uniform or non-uniform temperature, respectively. To find the segmental time of flight, for all but the 1st segment, the TOF through the $i$-th segment at the reference conditions, $t_{o f_{i}}\left(T_{r e f}\right)$ (appear in equation 21), is calculated as a value that maximizes the envelope cross-correlation of the echoes produced by echogenic features that bound the segment. This approach cannot be used for the first segment because the shape of the "initial bang" (occurs close to $t=0$ in Fig. 2) is too dissimilar from the waveform of the first echo. Instead, $t_{o f_{1}}\left(T_{r e f}\right)$ was found as $2 z_{1} / c$, where $z_{1}$ is listed in Table 1 and $c$ is the speed of sound at the reference temperature found during calibration experiments.

\subsection{Impact of thermal expansion}

The velocity of the ultrasound propagation changes with temperature. The thermal expansion, which changes length of the ultrasound propagation, is another factor that influences the return delays of ultrasound echoes as a function of temperature. The explicit contribution of these two factors to the measured time of flight is given by the following expression:

$$
t_{o f}(T)=\frac{2 L_{r e f}}{c(T)}+\frac{2 \alpha L_{r e f} \Delta T}{c(T)}
$$


where $L_{r e f}$ is the length of the ultrasound propagation at the reference conditions, and the second term in the right hand side describes the effect of the thermal expansion. For small $\Delta T=T-T_{r e f}$, the contribution of thermal expansion is negligible. As the deviation from the reference temperature increases, so does the contribution of the thermal expansion to the measured arrival delays.

In the current study, the correlation between the speed of sound and the temperature (the calibration curve of the method) was obtained experimentally by holding the waveguide at a known temperature and measuring the delay in return echoes from which the corresponding speed of sound is calculated. The red line in Fig. 5(a) shows the calibration curve that accounts for thermal expansion. It was obtained by using the measured $t_{o f}$ to calculate the speed of sound as

$$
c=(1+\alpha \Delta T) \frac{2 L_{r e f}}{t_{o f}(T)}=(1+\alpha \Delta T) c_{a}=f(T)
$$

The blue line in the same figure was obtained by ignoring the contribution of the thermal expansion and calculating an apparent speed of sound $c_{a}(T)$ as:

$$
c_{a}=\frac{2 L_{r e f}}{t_{o f}(T)}=f_{a}(T)
$$

The difference between the two curve is exactly the correction for the thermal expansion $\alpha \Delta T c_{a}$ ignored in (26).

When the temperature of the waveguide is unknown and the measured $t_{o f}$ is used to estimate it, we are presented with two choices:

(a) Either use $t_{o f}$ to calculate $c$ from equation (25) and then use the calibration curve compensated for thermal expansion (red line in Fig. 5(a)) to find $T$; or

(b) Calculate $c_{a}$ from equation (26) and find $T$ using the calibration that has not been compensated for the thermal expansion (blue line).

As illustrated in the insert of Fig. 5(a), the estimated temperature is the same in both cases. In essence, the error introduced by ignoring thermal expansion when calculating an apparent speed of sound is exactly compensated by the calibration curve obtained under the same approximation. Therefore, as long as the unknown temperature is uniformly distributed or the piecewise-constant segmental temperature distribution is used to reconstruct spatially varying $T(z)$, there is no practical need to remove the contribution of the thermal expansion to the change in the TOF with temperature and option (b) can be used without penalty on the accuracy of the obtained piecewise-constant temperature estimation.

The experimental results reported in this paper were obtained by interpreting the ultrasound delay measurement according to option (b). This option will generally introduce estimation errors when $T(z)$ is reconstructed under an assumption other than a piecewise-constant parameterization. With reference to the segmental time of flight (7), note that, as the density of the waveguide segmentation is increased, the temperature change within the segment will become smaller, which will reduce the relative change in the positions $z_{i}$ and $z_{i-1}$ due to temperature non-uniformity and the associated error introduced by ignoring it.

\section{Results}

\subsection{Calibration}

The correlation between the speed of sound and the temperature was obtained for the 4th segment of the waveguide located between its distal end and the echogenic feature at $z_{3}$ (Fig. 2). This segment was placed in the middle of the tube furnace (Fig. 4), where the temperature is uniform. The speed of sound through this segment was calculated using the following adaptation of equation (19):

$$
c_{4}=\frac{2 L_{4}}{\left(t_{o f_{4}}\left(T_{r e f}\right)-t_{o f_{3}}\left(T_{r e f}\right)\right)+\left(\Delta t_{o f}^{z_{4}}(T)-\Delta t_{o f}^{z_{3}}(T)\right)} .
$$

where $\Delta t_{o f}^{z_{3}}(T)$ and $\Delta t_{o f}^{z_{4}}(T)$ were determined following the described signal analysis procedure. The length of the segment $L_{4}=z_{4}-z_{3}$ was either assumed constant and defined by $z_{i}$ 's given in Table 1, or was adjusted by a factor $(1+\alpha \Delta T)$ to account for the thermal expansion, where $\alpha=4.3 \times 10^{-6} /{ }^{\circ} \mathrm{C}$ according to the manufacturer. The 


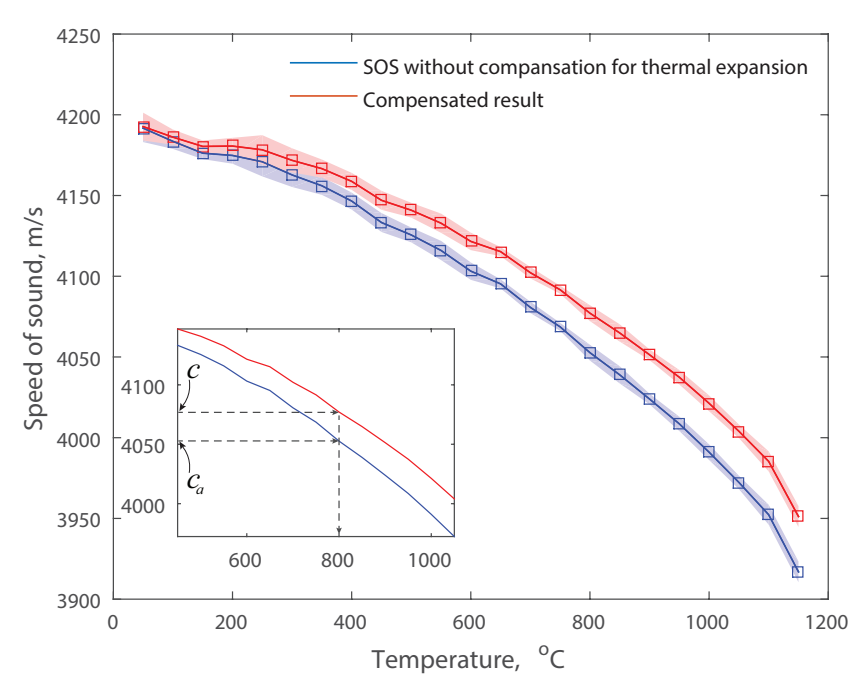

(a)

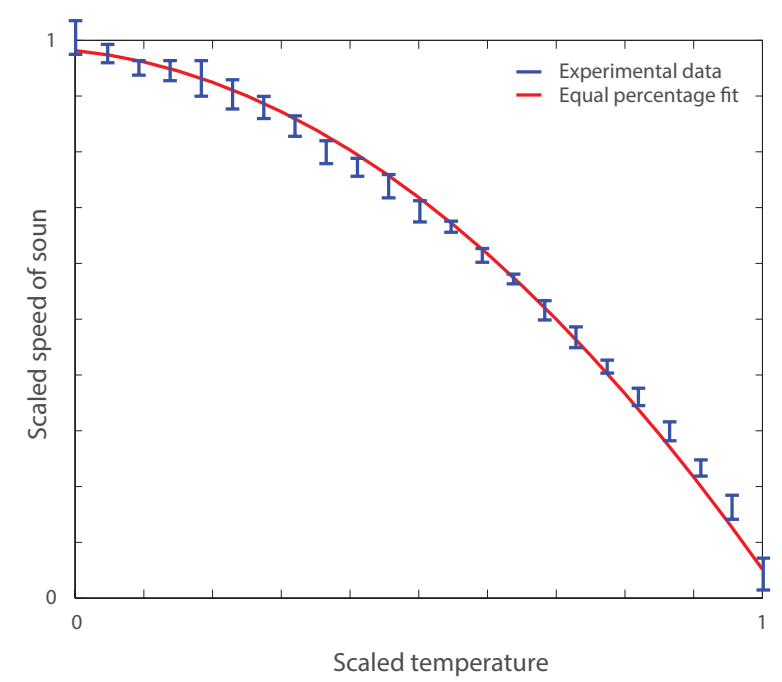

(b)

Figure 5: (a) Speed of sound before and after compensation for the thermal expansion is shown as an average of 6 separate calibration experiments. Shaded areas indicate $95 \%$ confidence interval. (b) The equal percentage fit (solid line) of the normalized speed of sound without compensating for the thermal expansion as a function of the normalized temperature. The error bars indicate $95 \%$ confidence interval for the normalized data.

TOF measurements at each temperature were repeated 6 times in random order. At each test temperature during the calibration experiments, in addition to waveform averaging performed internally by the oscilloscope, the collection of 20 waveforms was triggered and the averaged result was used in signal analysis to determine the speed of sound at a given temperature. Fig. 5(a) depicts the obtained speed of sound as a function of temperature with and without compensation for thermal expansion; the shaded area in this figure indicates the $95 \%$ confidence interval.

It is desirable to fit the experimental data into a functional form describing the relationship between the speed of sound and the temperature. Such functional dependence simplifies the interpretation of the TOF measurements. It also accelerates the process of obtaining the calibration curve for each new waveguide because fewer measurements are needed to find parameters defining such dependence.

The examination of the data in Fig. 5(a) reveals that a fixed increment in the temperature results in an approximately equal, in percentage terms, decrease in the speed of sound. We, therefore, selected the following functional form which captures the observed "equal percentage" dependence in the relative change of the speed of sound with temperature:

$$
\widetilde{c}=a-R^{(\widetilde{T}-b)}
$$

where $\widetilde{c}$ and $\widetilde{T}$ are normalized to change between 0 and 1 :

$$
\begin{aligned}
\widetilde{c} & =\frac{c(T)-c_{\min }}{c_{\max }-c_{\min }} \\
\widetilde{T} & =\frac{T-T_{\min }}{T_{\max }-T_{\min }}
\end{aligned}
$$

The values of the regression coefficients

$$
a=1.172, \quad b=0.943, \quad R=4.907
$$

provide the best least squares fit to the experimental data. The goodness of this fit was characterized by the coefficient of determination, which was found to be equal to $R^{2}=0.999$. Visual inspection of Fig. 5(b) confirms an excellent agreement between the obtained model (28) and the experimental data. After obvious manipulations, the obtained functional relationship was written as

$$
c(T)=c_{\text {min }}+\left(a-R^{(\widetilde{T}-b)}\right)\left(c_{\text {max }}-c_{\text {min }}\right)
$$




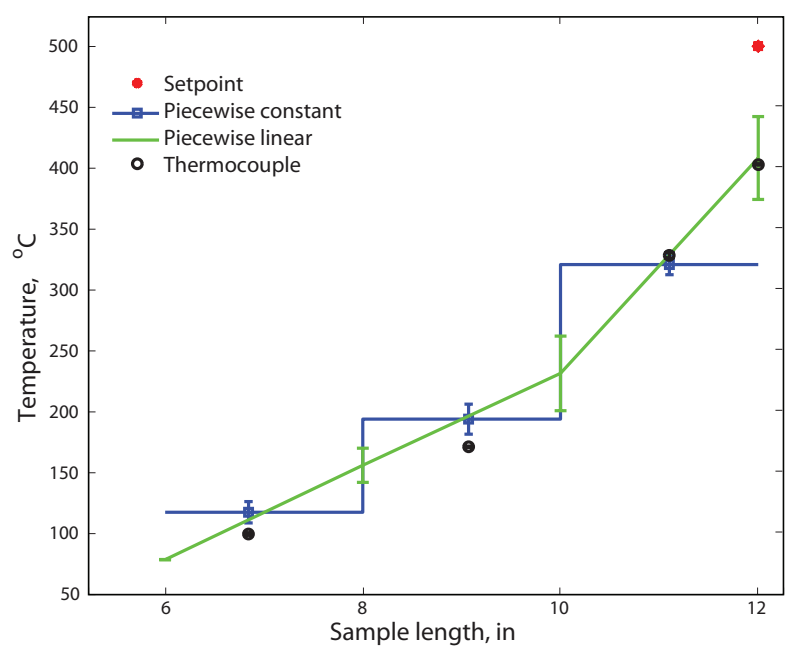

(a)

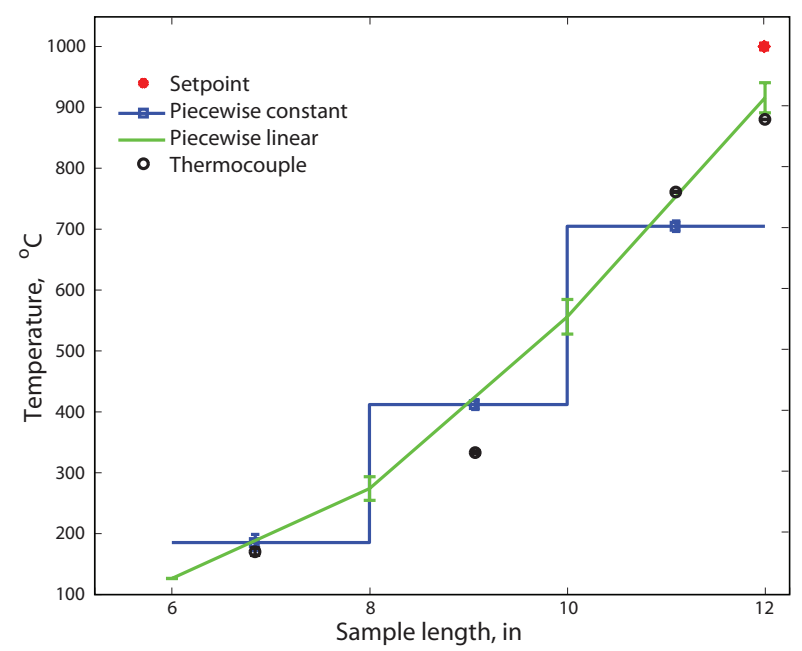

(b)

Figure 6: The estimated temperature distributions at the steady state. Panels (a) and (b) show the results when the setpoint of the furnace (red dots) was maintained either $500^{\circ} \mathrm{C}$ or $1,000^{\circ} \mathrm{C}$, respectively. The temperature distributions in the three segments of the waveguide were obtained using piecewise-constant and piecewise-linear parameterizations. For comparison, thermocouples were used to measure the surface temperatures (black dots) in the middle of the three segments and at the distal end of the waveguide.

where $c_{\min }$ and $c_{\max }$ correspond to the speed of sound at $T_{\max }=1,150^{\circ} \mathrm{C}$ and $T_{\min }=50^{\circ} \mathrm{C}$.

\subsection{Estimation of temperature distribution, $T(z)$}

The proposed method was applied to the measurement of non-uniform temperature distribution. This distribution was established by inserting the segment of the waveguide between its distal end $\left(z_{4}\right)$ and the last echogenic feature (located at $z_{3}$ ) into the entrance of the tube furnace and thermally insulating the remainder of the waveguide protruding outside the furnace by wrapping it with fiberglass insulation $\sim 2 \mathrm{~cm}$ thick. Two sets of experiments were conducted with the furnace setpoint maintained by the PID temperature controller at either 500 or $1,000{ }^{\circ} \mathrm{C}$. The experiments at the two different setpoints were repeated three times in a random order. During each experiment, an ultrasound excitation pulse was applied to the sample and the four return echoes were acquired after the thermocouple measurements stabilized at constant values. The data collection procedure was similar to the one used to obtain the calibration data and involved the averaging of 20 waveforms, which were acquired in a continuous succession, prior to using the average waveform sequence in signal analysis.

For the two sets of experiments, Figure 6 shows the furnace setpoint (red dots) and the corresponding measurements provided by the thermocouple attached to the distal end of the waveguide (black dots at $z_{4}$ ). Note that the heat loss to the environment and the conductive heat transfer through the waveguide, partially inserted into the furnace, resulted in a substantial difference between the setpoint values and the measured temperature, which was $\sim 400{ }^{\circ} \mathrm{C}$ when the furnace setpoint was set to $500^{\circ} \mathrm{C}$ and $\sim 880{ }^{\circ} \mathrm{C}$ for the $1,000{ }^{\circ} \mathrm{C}$ setpoint.

The segment $L_{1}$ of the waveguide between $z_{0}$ (transducer location) and $z_{1}$ (location of the first echogenic feature) was used as the delay line, for which the temperature estimation was not attempted. The change in the time of flight of all echoes, $\Delta t_{o f}^{z_{i}}(T)$, relative to the reference temperature of $20^{\circ} \mathrm{C}$ was calculated using the envelope cross-correlation method and the obtained values were used in the left-hand-side of equation (22) to calculate the corresponding value of the segmental time of flight, $t_{o f_{i}}(T)$. The segmental time of flight, the calibration curve (32) uncompensated for thermal expansion, and different parameterizations of the segmental temperature distribution were then used to estimate $T(z)$ between $z_{1}$ and $z_{4}$. For each parameterization, the parameters defining the temperature distribution were selected to satisfy equation (21) exactly. The results of the repeat experiments were averaged and the $95 \%$ confidence intervals were calculated for the averaged temperature estimation.

Piecewise constant distribution. The assumption of constant temperature in each segment of length $L_{i}$, coupled with the calibration Eqn. (32), gives the piecewise constant estimation of $T(z)$, with the temperature in the $i$-th segment 
Table 2: Piecewise linear distribution of segmental temperature

\begin{tabular}{c|c|c||c|c}
\hline & \multicolumn{2}{|c||}{ Furnace setpoint at $500{ }^{\circ} \mathrm{C}$} & \multicolumn{2}{c}{ Furnace setpoint at $1,000{ }^{\circ} \mathrm{C}$} \\
\hline & $m\left({ }^{\circ} \mathrm{C} / \mathrm{m}\right)$ & $n\left({ }^{\circ} \mathrm{C}\right)$ & $m\left({ }^{\circ} \mathrm{C} / \mathrm{m}\right)$ & $n\left({ }^{\circ} \mathrm{C}\right)$ \\
\hline$L_{2}$ & $1,552.91$ & -161.21 & $2,820.78$ & -308.24 \\
\hline$L_{3}$ & $1,524.64$ & -155.45 & $5,400.15$ & -833.15 \\
\hline$L_{4}$ & $3,414.09$ & -633.48 & $6,572.58$ & $-1,129.79$ \\
\hline
\end{tabular}

equal to

$$
T_{i}=\left(T_{\text {max }}-T_{\text {min }}\right) \frac{\ln \left(1+A-\frac{2 L_{i}}{c_{\text {min }} t_{o f_{i}}}\right)-\ln B}{\ln R},
$$

where

$$
\begin{aligned}
& A=\frac{a\left(c_{\max }-c_{\min }\right)}{c_{\min }},
\end{aligned}
$$

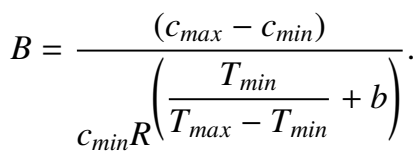

The obtained piecewise-constant temperature distribution between $z_{1}$ and $z_{4}$ is shown in Figure 6. For comparison, this figure also shows independent thermocouple measurements of the surface temperature in the middle of each segment. Though this simplest parametrization of the segmental temperature is characterized by physically infeasible discontinuity in the estimated temperature distribution at the location of echogenic features, it nevertheless correctly captures the trend in the temperature along the length of the sample.

Piecewise linear distribution. The piecewise linear segmental temperature distribution (9) was used in the correlation (32) and the result was substituted into equation (7). After integration, the following expression for the time of flight through the $i$-th segment of the waveguide is obtained:

$$
t_{o f_{i}}(T)=\left.\frac{2\left(T_{\min }-T_{\max }\right) \ln \left[c_{\min }+\left(c_{\max }-c_{\min }\right)\left(a-R^{\left.\left.\frac{b\left(T_{\min }-T_{\max }\right)-T_{\min }+m_{i} z+n_{i}}{T_{\max }-T_{\min }}\right)\right]+2 m_{i} z \ln R}\right.\right.}{m_{i}\left(\ln R+\ln \left[a\left(c_{\max }-c_{\min }\right)+c_{\min }\right]\right)}\right|_{z_{i-1}} ^{z_{i}}
$$

An additional condition needed to find unknown coefficients $m_{i}$ and $n_{i}$ is obtained by requiring that the temperature remains continuous at the boundary of two adjacent segments:

$$
m_{i} z_{i}+n_{i}=m_{i-1} z_{i}+n_{i-1}
$$

If we assume that $T(0)$ is measured independently (as was done in our experiments), giving $n_{1}=T(0)$, then the combination of equations (36) and (37) for all segments provides us with sufficient conditions to calculate $m_{i}$ and $n_{i}$ for all segments and the piecewise linear temperature distribution across the entire waveguide.

For the three segments of the waveguide, in which the temperature distribution was estimated, the described procedure results in the piecewise linear temperature distribution depicted in Fig. 6 and summarized in Table 2. The temperature discontinuity at the boundary of the segments is eliminated, and an improved agreement with the thermocouple measurements is apparent. For the segments $L_{2}$ and $L_{3}$ located outside the furnace, note higher deviation between the ultrasound measurements the thermocouple measurements. It reflects a heat loss through the cylindrical surface of these segments that lowers the surface temperature measured by the thermocouples relative to the core temperature at the waveguide's centerline measured by the US-MSTD method. 


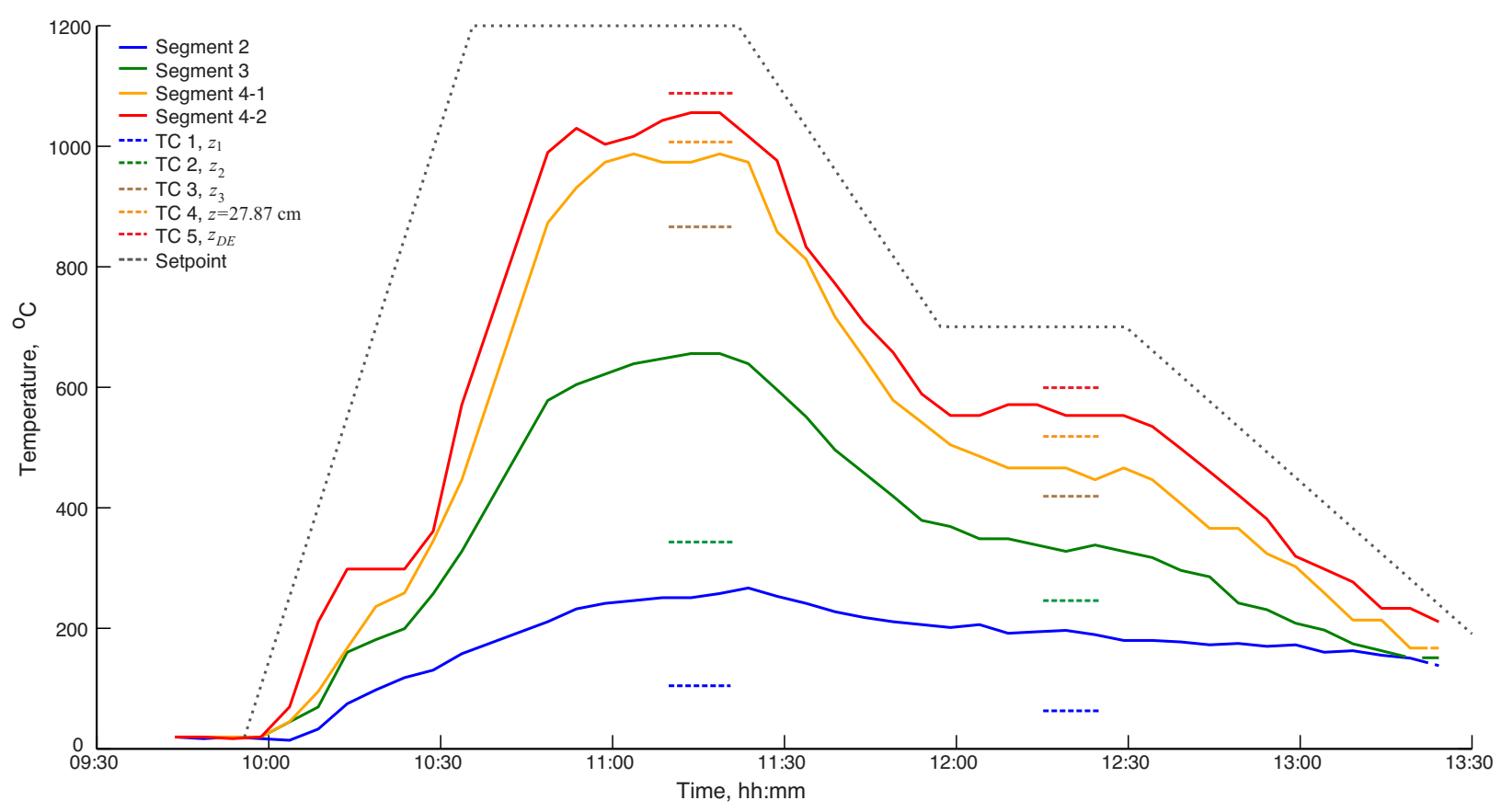

Figure 7: Real-time ultrasound measurements of the temperature distribution in the sample during transient changes in the furnace setpoint. Thermocouple measurements were logged manually after steady state was reached after each change in the setpoint.

\subsection{Measurements of temporal changes in temperature distribution, $T(z, t)$}

Preceding results were obtained with the furnace operated at a constant setpoint for the duration of each experiment. In order to validate the ability of the US-MSTD method to measure time-dependent temperature distribution, the setpoint was changed from zero (corresponds to room temperature) to $1,200^{\circ} \mathrm{C}$, and then reduced first to $700{ }^{\circ} \mathrm{C}$ and back to room temperature. The described temporal trajectory of the setpoint is shown as a dotted black line in Fig. 7.

Prior to time-varying temperature experiments, the alumina waveguide was modified by adding an additional echogenic feature by drilling an additional 3/32" hole approximately one inch away from the distal end. The purpose of this modification was to demonstrate an improved spatial resolution of segmental temperature reconstruction achieve with the finer segmentation of the waveguide. The procedure described in section 3.1 was used to precisely localize this additional echogenic feature as being located at $z=27.87 \mathrm{~cm}$. As a result of this modification, $L_{4}$ segment of the waveguide was subdivided into two approximately 1"-long sub-segments, referred to as segments 4-1 and 4-2 in Fig. 7. The placement of the thermocouples was changed to coincide with the locations of the four echogenic features. As before, an additional thermocouple was attached to the distal end of the waveguide. The measurements from all thermocouples were logged only after the measurements stabilized at new setpoint values, as shown in Fig. 7.

As the setpoint transitioned following the selected temporal trajectory, the ultrasound measurements of the segmental time of flights were acquired every $5 \mathrm{~s}$ throughout the duration of the experiment. The obtained time-dependent estimation of the piecewise constant temperature distribution is shown in Fig. 7. The expected smaller spatial variation in the temperature across short segments 4-1 and 4-2, located inside the heating zone, and a rapid temperature decrease in the temperature along the sections protruding outside the furnace, where thermal losses to the environment are high, can be observed. Thermal losses are also responsible for the observed difference between the thermocouple measurements that are more reflective of the surface temperature of the waveguide and the ultrasound measurements that respond to changes in its core temperature. The measurements of all segmental temperatures are consistent with the prescribed evolution of the temperature setpoint and with the independent thermocouple measurements of the surface temperature at steady state conditions. It demonstrates that the developed method can be effectively used to measure temperature distribution in real time during dynamic transitions over a wide range of operating conditions. 


\section{Conclusions}

We have developed and experimentally demonstrated a new method for non-invasive continuous ultrasound measurements of the temperature distribution in solids. By segmenting the ultrasound propagation path, the proposed approach is capable of monitoring temperature distribution with higher accuracy and lower uncertainty inside solid structures, such as refractories and other process containments. The ability to measure the steady state $T(z)$ and the time varying temperature distribution $T(z, t)$ was demonstrated. Therefore, this approach can be used to continuously measure the changing temperature distribution over a large range of values, and is particularly useful when the measurements are needed to characterize extreme environments in which the performance of conventional sensors is quickly degraded, or when the deployment of traditional insertion probes is difficult, impossible or undesirable.

The method uses the measurements of the segmental time of flight of multiple ultrasound echoes to infer the temperature distribution in different segments of the solid sample by solving an integral measurement equation. To enforce the uniqueness of the solution, the unknown temperature distribution must be constrained (parameterized) to take an allowable form. Three parameterizations were discussed in this paper (piecewise constant, piecewise linear, and the parametrization with the thermal conductivity model), two of which were used in experimental testing. Unlike the previous demonstration [13], where only a piecewise constant parameterization was used, the current implementation of the method avoids infeasible discontinuities in the estimated temperature distribution when a piecewise linear parametrization is used.

Previous demonstration of the method [13] was for relatively low temperatures that changed within $100^{\circ} \mathrm{C}$ range along the length of a cementitious sample and a piecewise constant approximation of an unknown temperature distribution. The narrow range of temperatures justified the assumption of the linear correlation between the temperature and the velocity of the ultrasound propagation. In this paper, the US-MSTD method is implemented and successfully tested for the wide range of temperatures that in some experiments exceeded $1,000^{\circ} \mathrm{C}$. Because the linear approximation no longer holds for the range of temperatures this wide, the method was implemented after fitting the data on temperature dependance of the speed of sound to a non-linear (exponential) model.

This and the previous demonstration of the method utilize longitudinal (primary) waves. Since the propagation velocity of all elastic waves is temperature dependent, the US-MSTD method may be extended to utilize other types of waves (e.g., s-waves) or their combination. The developed method may also be applied to characterize segmental distribution of a property other than temperature that influences the speed of ultrasound propagation, such as a sample's density or its elasticity.

\section{Acknowledgements}

The authors acknowledge financial support from the U.S. Department of Energy's National Energy Technology Laboratory, under award number DEFG2611FE0006947.

\section{References}

[1] S. F. Green, An acoustic technique for rapid temperature distribution measurement, Journal of the Acoustical Society of America 77 (2) (1985) $759-763$

[2] Y. J. Lee, B. Khuri-Yakub, K. Saraswat, Temperature measurement in rapid thermal processing using the acoustic temperature sensor, Semiconductor Manufacturing, IEEE Transactions on 9 (1) (1996) 115 -121

[3] R. Arthur, W. Straube, J. Trobaugh, E. Moros, Non-invasive estimation of hyperthermia temperatures with ultrasound, International journal of hyperthermia 21 (6) (2005) 589-600.

[4] H. Zhang, M. Basin, M. Skliar, Itó-volterra optimal state estimation with continuous, multirate, randomly sampled, and delayed measurements, IEEE Trans. Automat. Control 52 (2007) 401-416.

[5] S. T. J Lu, K Wakai, S. Shimizu, Acoustic computer tomographic pyrometry for two-dimensional measurement of gases taking into account the effect of refraction of sound wave paths, Measurement Science and Technology 11 (6) (2000) $692-697$.

[6] B. Praher, K. Straka, G. Steinbichler, An ultrasound-based system for temperature distribution measurements in injection moulding: system design, simulations and off-line test measurements in water, Measurement Science and Technology 24 (8) (2013) 084004.

[7] A. Kosugi, I. Ihara, A simple method for profiling surface temperature distributions by laser-ultrasound, Journal of Solid Mechanics and Materials Engineering 5 (12) (2011) 702-708.

[8] A. Balakrishnan, J. Lions, State estimation for infinite-dimensional systems, Journal of Computer and System Sciences 1 (1967) 391 - 403.

[9] M. Takahashi, I. Ihara, Ultrasonic determination of temperature distribution in thick plates during single sided heating, Modern Physics Letters B 22 (11) (2008) 971-976. 
[10] P. L. Schmidt, D. G. Walker, D. J. Yuhas, M. M. Mutton, Thermal measurements using ultrasonic acoustical pyrometry, Ultrasonics 54 (4) (2014) 1029-1036.

[11] R. Ramalingam, H. Neumann, Fiber bragg grating-based temperature distribution evaluation of multilayer insulations between 300 kÂUU-77 k, Sensors Journal, IEEE 11 (4) (2011) 1095-1100.

[12] P. A. Hanscombe, P. N. Richards, Distributed temperature sensor, uS Patent 5,286,109 (Feb. 15 1994).

[13] Y. Jia, M. Puga, A. E. Butterfield, D. A. Christensen, K. J. Whitty, M. Skliar, Ultrasound measurements of temperature profile across gasifier refractories: Method and initial validation, Energy and Fuels 27 (8) (2013) 4270-4277.

[14] M. Skliar, K. Whitty, A. Butterfield, Ultrasonic temperature measurement device, US Patent 8,801,277 B2, (2014).

[15] M. Parrilla, J. J. Anaya, C. Fritsch, Digital signal processing techniques for high accuracy ultrasonic range measurements, IEEE Transactions on Instrumentation and Measurements 40 (4) (1991) 759-763.

[16] D. Hull, H. Kautz, A. Vary, Measurement of ultrasonic velocity using phase-slope and cross-correlation methods, Materials evaluation 43 (11) (1985) 1455-1460.

[17] C. de Korte, A. van der Steen, B. Dijkman, C. LancÃl'e, Performance of time delay estimation methods for small time shifts in ultrasonic signals, Ultrasonics 35 (4) (1997) 263 - 274.

[18] G. Jacovitti, G. Scarano, Discrete time techniques for time delay estimation, IEEE Transactions on Signal Processing 41 (2) (1993) $525-533$.

[19] F. Viola, W. Walker, A comparison of the performance of time-delay estimators in medical ultrasound, IEEE Transactions on Ultrasonics, Ferroelectrics, and Frequency Control 50 (4) (2003) 392-401.

[20] B. Champagne, E. Eizenman, S. Pasupathy, Exact maximum likelihood time delay estimation for short observation intervals, IEEE Transactions on Signal Processing 39 (6) (1991) 1245-1257.

[21] L. H. Le, An investigation of pulse-timing techniques for broadband ultrasonic velocity determination in cancellous bone: a simulation study, Physics in Medicine and Biology 43 (8) (1998) 2295 - 2308.

[22] Y. Jia, M. Skliar, Anisotropic diffusion filter for robust timing of ultrasound echoes, in: Ultrasonics Symposium (IUS), 2014 IEEE International, IEEE, 2014, pp. 560-563. 